\title{
Improving the Prediction of Response to Therapy in Autism
}

\author{
Stephen Bent* ${ }^{* *}$ and Robert L. Hendren* \\ ${ }^{*}$ Department of Psychiatry, ${ }^{\dagger}$ Osher Center for Integrative Medicine, Department of Medicine, and ${ }^{\ddagger}$ Department of Epidemiology \\ and Biostatistics, University of California, San Francisco, San Francisco, California 94121
}

\begin{abstract}
Summary: Autism is a heterogeneous disorder involving complex mechanisms and systems occurring at diverse times. Because an individual child with autism may have only a subset of all possible abnormalities at a specific time, it may be challenging to identify beneficial effects of an intervention in double-blind, randomized, controlled trials, which compare the mean responses to treatments. Beneficial effects in a small subset of children may be obscured by the lack of effect in the majority. We review the evidence for several potential model systems of biochemical abnormalities that may contribute to the etiology of autism, we describe potential biomarkers or treatment targets for each of
\end{abstract}

these abnormalities, and we provide illustrative treatment trials using this methodology. Potential model systems include immune over and under reactivity, inflammation, oxidative stress, free fatty acid metabolism, mitochondrial dysfunction, and excitotoxicity. Including potential biomarkers and targeted treatments in clinical trials for autism provides a potential method for limiting the heterogeneity of enrolled subjects, which may improve the power of studies to identify beneficial effects of treatments while also improving the understanding of the disease. Key Words: Autism, randomized controlled trials, biomarker, complementary, and alternative therapy.

\section{INTRODUCTION}

Our understanding of the etiology of autism is growing rapidly, and as it grows it is changing the way we are considering interventions. Evidence is mounting that multiple genes are involved, their expression is influenced through environmental interaction, the immune system plays a role in many cases, and there are likely critical periods when the active process of epigenetic changes pushes the neurodevelopmental trajectory toward the autism spectrum. Evidence-based standards of intervention, such as behavioral therapies, speech and language interventions, and medications for troubling behavioral symptoms associated with autism are being joined with understudied nutritional and alternative "biomedical' interventions in many treatment programs. Designing clinical trials to evaluate the effectiveness of these nutritional and biomedical interventions is challenging and complicated. In this article, we provide several examples of these interventions, describe some of

Address correspondence and reprint requests to: Stephen Bent, M.D., Department of Medicine, Osher Center for Integrative Medicine, University of California, San Francisco, SFVAMC, 111-A1, 4150 Clement Street, San Francisco, CA 94121. E-mail: stephen.bent@ucsf.edu. the problems encountered in trial design, and offer potential solutions from our clinical trial experience.

\section{BACKGROUND}

Autistic spectrum disorders (ASDs) are now estimated to affect 1 in 110 children in the United States, ${ }^{1}$ signaling an even more urgent need to develop effective therapies for the millions of children affected by this disorder. Although there have been many advances in the awareness and understanding of ASDs, the volume of scientific evidence evaluating the safety and efficacy of interventions (both behavioral and pharmacological) is relatively small. For example, behavioral and educational interventions are considered to be the standard of care for children with ASD, and an expert committee from the Institute of Medicine has recommended that affected children receive of individualized educational therapy for a minimum of 25 hours per week, 12 months per year. ${ }^{2}$ But even for behavioral interventions, the evidence for efficacy from high-quality randomized, controlled trials is quite limited. In a recent systematic review that identified 101 studies evaluating the efficacy of behavioral and developmental interventions for ASD, the median sample size of studies was only 22 , and there were numerous methodological problems with the majority of studies, 
including inadequate description of blinding, lack of description of concealing the allocation sequence, and limited or no description of the technique of randomization. ${ }^{3}$ The review concluded that while the evidence suggests that the Lovaas method of applied behavioral analysis "may improve some core symptoms of ASD compared to special education," the findings were "based on pooling outcomes from a few, methodologically weak studies." 3 Furthermore, no evidence from randomized, controlled trials was available to compare the efficacy of the different early intervention programs.

In regard to pharmacological therapies, only two drugs have been approved by the Food and Drug administration for specific indications in children with autism, and neither has been shown to improve the core features of the disorder. Risperidone, an atypical antipsychotic, was approved from the treatment of irritability associated with autism. The approval was largely based on the findings from two randomized, controlled trials in 79 and 101 children with autistic disorder and severe behavioral problems. ${ }^{4,5}$ In the larger study, children in the risperidone group had a $57 \%$ reduction in irritability compared to only a $14 \%$ reduction among children in the placebo group $(p<0.001) .{ }^{4} \mathrm{~A}$ second atypical antipsychotic, aripiprazole, was approved in late 2009 for the same indication (irritability) in children with ASD, based on the results from two 8-week randomized, placebo-controlled trials in 98 and 218 patients. ${ }^{6,7}$ In the larger study, irritability improved $14 \%$ to $21 \%$ over 8 weeks, but side effects, including sedation, tremor, drooling, and weight gain, were common. ${ }^{6}$ The long-term safety of both agents in this population is not yet established.

Two larger randomized, controlled trials of commonly used drug therapies in children with ASD reported negative results in 2009. In a study of 149 children aged 5 to 17 with ASD, citalopram was found to be no better than placebo for improving the "overall severity" of autism, as measured by the Clinical Global Impression (CGI) score $(32 \%$ positive response in the citalopram group compared to a $34 \%$ positive response in the placebo group; $p=0.99$ ). Children treated with citalopram in this study also had no greater improvement than a placebo in a standard measure of obsessive-compulsive symptoms. ${ }^{8}$ In another randomized, controlled trial $(\mathrm{n}=158)$, fluoxetine was found to be no better than a placebo for improving repetitive behaviors. ${ }^{9}$ The full details of this study have yet to be published.

For most nutritional and biomedical therapies, there is considerably less evidence than for behavioral or pharmacological interventions. While recent surveys have found that as much as $95 \%$ of families have used or are currently using nutritional or biomedical therapies to treat their affected child, ${ }^{10}$ the vast majority of these interventions have not been examined with even a single, high-quality randomized, controlled trial.

\section{CHALLENGES TO IDENTIFYING EFFECTIVE THERAPIES FOR AUTISM}

As new studies are designed to evaluate the efficacy of both currently used treatments and novel therapies, it will be crucial to focus on the methodology of the randomized, controlled trials in autism to optimize the ability of these studies to identify a beneficial effect, if one truly exists, and to determine which patients with ASD are most likely to benefit. There are a number of unique challenges to identifying and evaluating specific therapies that may be helpful for improving symptoms of ASD. These include the heterogeneity of the disorder, the large number of symptoms that may be selected as targets for the therapy, and the challenges in enrolling this population in clinical trials. As follows, we discuss these problems in more detail, and we suggest some possible solutions for improving clinical trials evaluating the efficacy of interventions in ASD.

\section{HETEROGENEITY OF AUTISTIC SPECTRUM DISORDERS}

ASDs are appropriately named because they truly do encompass a "spectrum" of clinical findings. Although there are three "core" features of autism (communication problems, social difficulties, and repetitive/restricted behaviors), these symptoms vary markedly both within and between individuals. Some authors have recommended the use of the term "autisms" rather than "autism" to highlight the fact that each child has a unique constellation of symptoms that likely reflects different underlying genetic, structural, and physiological characteristics. In addition, many children have varying degrees of associated symptoms, including gastrointestinal problems, comorbid psychiatric symptoms such as anxiety or obsessive compulsive symptoms, and auditory processing or sensory abnormalities. Similarly, symptoms of ASD change markedly as children age, and improvement may be related to a variety of factors that are not always observed or measured in clinical trials. The implication of this heterogeneity within and between children with ASD is that it is difficult to recruit a population of children with similar symptoms that will be stable during the course of the study (except for changes in symptoms that are due to the intervention). The increased variability of baseline measures results in the statistical problem of a larger, standard deviation of any symptom score, and thus there is more "noise" and less power to identify beneficial effects. One approach to this problem is to conduct larger studies. However, it has been difficult to recruit large numbers of children of ASD for interventional studies, and these studies are still limited by the standard analytical technique of comparing mean re- 
sponses in all children, when only certain subsets of children may be most likely to respond to treatment.

The heterogeneity of ASD may be explained by one or several underlying biochemical mechanisms that contribute to symptoms within an individual. Although it is difficult to know whether abnormal biochemical signals are the cause or the effect of the ASD of the individual, knowledge of these potential biomarkers may allow for more targeted treatment studies and tailoring of therapies to individuals with specific abnormalities. As follows, we briefly describe some of these areas of potential biochemical abnormalities, and we then present some initial pilot studies that include these biomarkers, both as a means of understanding the disorder and assessing response to therapy.

\section{POTENTIAL MODEL SYSTEMS FOR UNDERSTANDING ABNORMAL PHYSIOLOGY IN AUTISM}

Recent research suggests that there may be new treatment targets that identify subgroups of people with autism based on epigenetic mechanisms rather than symptoms. As follows, we briefly describe some of these potential mechanisms and targets and the literature that is developing regarding their suitability for clinical trials.

\section{Immune over and under reactivity}

Increasing evidence suggests that the immune system in children with ASD is altered. Studies have found reduced levels of the plasma $\operatorname{IgG}$ and $\operatorname{IgM}$ in children with autism compared to controls, ${ }^{11}$ altered monocyte responses to stimulation, ${ }^{12}$ and reduced cytotoxicity of natural killer cells. ${ }^{13}$ Other studies have documented antibodies directed against fetal brain tissue, ${ }^{14}$ suggesting a possible mechanism for immune dysfunction, leading to alterations in early brain development. Altered immune responses may also affect the inflammatory state of the child's body, which could affect both normal development and the severity of specific symptoms.

\section{Inflammation}

Immune dysfunction and inflammation are integrally related, and many of the studies previously noted have also documented abnormal levels of inflammatory markers, which were most commonly the plasma cytokines. Children with ASD have been noted to have increased levels of the pro-inflammatory cytokines MCP-1, interleukin (IL)-6, interferon- $\alpha$, INF- $\gamma$ and tumor necrosis factor- $\alpha$ (TNF- $\alpha$ ) in the brain or in the circulating blood. ${ }^{15-19}$ Inflammation may mediate specific symptoms in ASD. For example, children with ASD and gastrointestinal symptoms were noted to have increased T-lymphocyte production of the pro-inflammatory cytokines, TNF- $\alpha$, and INF- $\gamma$ but decreased T-lymphocyte production of the anti-inflammatory cytokine, IL-10 in colonic, upper and lower small intestinal tissue. ${ }^{20}$

\section{Oxidative stress}

Oxidative stress refers to an increased level of free radicals from oxygen, nitrogen, and sulfur molecules (often abbreviated as reactive oxygen species [ROS]). The ROS are highly reactive, and while they have important roles in cell signaling, apoptosis, gene expression, and ion transport, an excess of ROS can lead to an attack on bases in nucleic acids, amino acid side chains in proteins, and double bonds in unsaturated fatty acids. These reactions may cause damage to DNA, RNA, proteins, and lipids, and an increased risk for cardiovascular disease, cancer, and possibly neurological diseases including ASD. A number of studies in children with autism have noted either increased levels of markers of oxidative stress or impaired capacity of children with ASD to manage ROS. Abnormal lipid peroxidation, as well as decreased levels of antioxidants, including glutathione, superoxide dismutase, transferrin, and ceruloplasmin have been reported in children with ASD. ${ }^{21-23} \mathrm{~A}$ case-control study in Egypt found that two markers (i.e., plasma F2-isoprostane and glutathione peroxidase) revealed increased levels of oxidative stress in $89 \%$ of children with ASD, and that the children with increased oxidative stress had higher odds of having antineuronal antibodies. $^{24}$ Another study found that an intracellular marker of the capacity to manage oxidative stress (the ratio between oxidized glutathione and oxidized disulfide glutathione) was reduced in lymphoblastoid cells of children with ASD. ${ }^{25}$ This marker of detoxification capacity also showed a greater decrease in response to a challenge with a pro-oxidant in children with ASD compared to control children. Impaired detoxification may be particularly problematic in children with ASD who have increased states of inflammation or other mechanisms that increase concentrations of ROS.

\section{Free fatty acid metabolism}

Neural tissue consists of approximately $60 \%$ lipids, the majority of which are phospholipids, and the most common phospholipids are the fatty acids, docosahexeanoic acid and arachidonic acid (ARA), which make up more than $20 \%$ of the brain dry weight. ${ }^{26}$ Recent evidence suggests that fatty acids are involved in the regulation of nerve signal transduction, neuro-inflammation, and cellular repair. ${ }^{26}$ Consequently, some authors have theorized that abnormal levels of specific free fatty acids may be involved in the pathogenesis or symptom burden of ASD. Three prior studies have reported low levels of omega-3 fatty acids in children with ASD compared to controls, ${ }^{27-29}$ whereas two found no difference. ${ }^{26,30}$ Thus far, there have been few consistent findings among prior studies to suggest a particular fatty acid deficiency. However, two of the studies found an increase in the ratio of 
ARA to eicosapentaenoic acid. ${ }^{26,27}$ The ARA:eicosapentaenoic acid ratio has been considered to be an "inflammatory index," because ARA produces compounds that lead to inflammatory responses, whereas the compounds derived from eicosapentaenoic acid have the opposite effects. Therefore, these preliminary studies suggest that abnormal fatty acid levels in children with ASD may be associated with increased inflammation.

\section{Mitochondrial dysfunction}

Mitochondrial dysfunction has gained attention in recent years as a possible cause of ASD or associated symptoms (see article by Bauman in this issue, page 320). Mitochondria are the main source of energy production for eukaryotic cells, which occurs along five enzyme complexes, each of which has several subunits. Mutations or deletion/duplication of the mitochondrial genome can result in impaired energy production. Several rare syndromes are known, each producing varying degrees of neurological dysfunction and associated problems with behavior, cognition, and motor function that may mimic symptoms of ASD. ${ }^{31}$ A population-based study in Portugal found that 5 of $69(7.2 \%)$ of children with autism had definite mitochondrial respiratory chain disorder dysfunction, and another $6(8.7 \%)$ had possible or probable mitochondrial disease. ${ }^{32}$ Perhaps most interesting is the fact that none of these children had any of the known mitochondrial DNA mutations (i.e., their suspected diagnosis of mitochondrial dysfunction was based on abnormal muscle biopsy or elevated lactic acid), suggesting that there may be unknown mitochondrial genetic abnormalities that produce symptoms of ASD. Furthermore, because known mitochondrial disorders are rare in the general population (occurring in 1 in 10,000 children), the increased prevalence in children with ASD suggests that these disorders may be related to disease pathogenesis. In a careful phenotypic and biochemical analysis of 25 children with ASD and known mitochondrial disorders, Weissman et al. ${ }^{33}$ noted that most children with ASD and mitochondrial dysfunction have findings that are not typical of idiopathic autism. Specifically, children with ASD and mitochondrial dysfunction had a very high prevalence of delayed gross motor development (64\%), and $40 \%$ had unusual patterns of regressive symptoms. Other evidence of organ dysfunction, including cardiac, hematologic, growth retardation, and fatigability were more common in these children. Nonetheless, the authors suggest that a broader clinical, biochemical, and genetic spectrum of mitochondrial disorders is likely to exist in ASD. ${ }^{33}$

\section{Excitotoxicity}

Children with ASD have an increased risk of seizures, which suggests a possible imbalance of excitatory and inhibitory neurotransmitters. Glutamate (the primary excitatory neurotransmitter in the brain) has been found to be elevated in the CSF of children with ASD. ${ }^{34}$ Preliminary studies have suggested that glutamate antagonists, such as memantine, may improve symptoms. ${ }^{35}$ Other studies have suggested that GABA, the primary inhibitory neurotransmitter in the brain, may be reduced in children with ASD. ${ }^{36}$ Reduced levels of GABA have been associated with hyperactivity, impaired sleep, seizures, mental retardation, and impaired motor coordination. ${ }^{37}$ In this issue of Neurotherapeutics, Wang et al. (page 264) discuss the important role of glutamate and the mGluR5 system and the downregulation of the GABA system in fragile $X$, and de Vries (page 275) discusses the upregulation of mammalian target of rapamycin as a treatment target for rapamycin in tuberous sclerosis.

\section{ADDRESSING HETEROGENEITY THROUGH THE USE OF BIOMARKERS}

A greater understanding of the potential abnormal physiological abnormalities in autism may help to inform the design of clinical trials of nutritional or biomedical interventions. If a particular intervention is designed to address a suspected physiological abnormality in ASD, then it may be desirable to recruit only the subset of patients who have clear evidence of that abnormality. If the suspected "target" of a biomedical intervention is not known, then it may be reasonable to collect data on several different possible biomarkers in early pilot studies to determine if beneficial effects are correlated with changes in these compounds. Selection on the basis of a likely biomarker may reduce heterogeneity in a study, and may provide greater power to observe a benefit if one truly exists. The potential disadvantage of this approach is that it may exclude certain subjects who could benefit from the intervention, so initial studies should consider using the biomarker to investigate mechanistic pathways rather than to exclude patients. Below, we will use examples of ongoing or recently completed studies to highlight the search for genes, inflammatory markers, oxidative states, and imaging features, which may isolate specific features of autism that are amenable to treatment with targeted therapies.

\section{Examples of using biomarkers to understand, identify, or predict response to therapy}

Oxidative stress: Methyl-B12. As previously noted, other previous studies have suggested that children with ASD may have increased levels of oxidative stress, possibly related to deficiencies in certain metabolic pathways. Two prior studies evaluated the metabolites in a specific biochemical pathway (the methionine cycle), and found that children with autism had abnormally low levels of reduced glutathione (GSH), which is the major intracellular antioxidant and mechanism for detoxifica- 
tion, and these children had abnormally high levels of the oxidized disulfide form of GSH (GSSG), which is a pattern that indicates increased oxidative stress. ${ }^{22,25}$ Furthermore, both studies found that supplementation with methyl B12 (methylcobalamin) improved the levels of these metabolites, indicating an improved capacity to manage oxidative stress. One of the studies also noted improvements in a standard scale of adaptive behavior (the Vineland Adaptive Behavior Scale), suggesting that the metabolic changes from supplementation might also lead to improvements in symptoms. ${ }^{25}$ However, this observation was limited because the study was open-label and uncontrolled. To test whether improving oxidative stress might benefit the symptoms associated with ASD, we designed a study to determine if methyl B12 treatment improves behavioral measures in children with autism and if improvement is associated with increased plasma concentrations of GSH and an increased redox ratio of reduced GSH to GSSG. ${ }^{38}$ The study was a 12 week, double-blind, placebo-controlled, crossover clinical trial of injectable methyl B12 at a dose of 64.5 $\mathrm{mcg} / \mathrm{kg}$ every 3 days (administered subcutaneously into the buttocks). Following this 12-week study, subjects were given the option of entering a 6-month open label trial of methyl B12. Subjects were 3 to 8 years old with autism, and received 6 weeks of a placebo and 6 weeks of methyl B12. Blood for GSH analysis and behavioral assessments were obtained at baseline, at week 6 , and at week 12 . Thirty subjects completed the 12 -week, doubleblind crossover study, and 22 subjects completed the 6-month extension study. No statistically significant differences in means of behavior tests or in glutathione status were identified between active and placebo groups. Nine (30\%) subjects demonstrated clinically significant improvement $(p<0.05)$ on the CGI scale and at least two additional behavioral measures that included Parent Interview for Autism (PIA-CV), Childhood Autism Rating Scale (CARS), Peabody Picture Vocabulary Test (PPVT-III), Stanford Binet Fifth Edition Routing Subsets, Aberrant Behavior Checklist (ABC), Child Behavior Checklist (CBCL), and the MacArthur Communication Developmental Inventory (MCDI). More notably, these responders exhibited significantly increased concentrations of GSH and GSH/GSSG ( $p=0.028)$. We concluded that an increase in glutathione redox status (GSH/ GSSG) may provide a biomarker for treatment response to methyl B12. ${ }^{38}$ Based on these findings, we have received funding to enroll 50 children with autism in a parallel group, placebo-controlled study of methyl B12 in which we will analyze the first 25 children to see if we can identify a biomarker (from the methionine to GSH pathway), which is related to treatment response. If identified, we will use this measure to screen and selectively enroll the next 25 children. If we do not find a significant biomarker, we will continue entering children in the study without screening and see if we can find a statistically reliable biomarker of response using the response in all 50 subjects.

\section{Genomics: Using genes to predict response to risperidone}

As previously noted, risperidone is one of only two approved drug therapies for the treatment of symptoms commonly associated with ASD, and it has been shown to substantially reduce irritability. ${ }^{4}$ However, even in a carefully recruited study population of children with severe tantrums, aggression, or self-injurious behavior, approximately one third of the enrolled children did not have a positive response to therapy. ${ }^{4}$ Because significant side effects (i.e., increased appetite, fatigue, drowsiness, dizziness, drooling) were noted with risperidone, and because the long-term safety of this drug in children is not known, it would be useful to have a more reliable method of identifying children who are most likely to benefit from therapy.

Genomic profiling of tissues has proven useful for classifying various malignancies and predicting both positive and negative treatment outcomes. ${ }^{39-45}$ Based on these studies and preliminary data, a pilot study was funded to demonstrate whether the efficacy, dose, and occurrence of side effects of risperidone for the treatment of serious behavioral symptoms in children with autism would be reflected in specific gene expression/RNA profiles in the blood prior to treatment. We recruited 50 children (4 to 18 years old) with autism who also have serious behavioral problems, and we treated them with risperidone. Blood samples were obtained prior to treatment and after 8 weeks of treatment. Treatment efficacy was assessed using the irritability subscale of the Aberrant Behavior Checklist (ABC) and the CGI-Improvement scale. In our preliminary analyses of this data, multiple genes associated with neuronal cell growth are associated with a positive response to risperidone ( $p=$ 0.001); a higher Autism Diagnostic Observation Schedule score is positively associated with body mass index; and higher body mass index is associated with genes related to cortisol and gastrointestinal metabolism. Further analysis of these and other associations are underway. It may be possible to identify specific genomic profiles that determine which children are most likely to benefit or experience a side effect before initiating therapy.

\section{Inflammatory markers: Omega-3 fatty acids}

Omega- 3 fatty acids are commonly used by families to attempt to improve symptoms in children with ASD. One recent survey found that $29 \%$ of children with ASD were currently using this supplement. ${ }^{46}$ One small pilot randomized, controlled trial $(n=13)$ found that omega-3 fatty acids led to a trend in the reduction of hyperactivity in children with ASD who were attending a day-care 
center, but this initial study did not include any assessments of a possible mechanism of action. ${ }^{47}$ Prior studies of omega-3 fatty acids in adults with coronary artery disease have indicated that the beneficial effects on heart disease may be due to reductions in inflammation, possibly through suppression of monocyte production of the cytokines IL- 1 and TNF- $\alpha{ }^{48}$ Omega-3 fatty acids are also believed to reduce oxidative stress, possibly through their ability to increase the activity of certain antioxidant enzymes, including xanthine oxidase and superoxide dismutase. ${ }^{49}$ Prior studies have found that supplementation with omega-3 fatty acids reduces the level of F2-isoprostanes, which is a marker of oxidant stress. ${ }^{49}$ Omega-3 fatty acids may therefore be considered as a potential treatment in children with elevated markers of oxidative stress. If omega- 3 fatty acids have a true beneficial effect on hyperactivity, it is also possible that this effect might be mediated through a change in the fatty acid levels in plasma or tissue. Three prior studies have reported low levels of omega- 3 fatty acids in children with ASD compared to controls, ${ }^{27-29}$ while two found no difference. $^{26,30}$

To expand on the early evidence suggesting a possible beneficial effect of omega-3 fatty acids in children with ASD, we conducted a larger randomized controlled trial of 27 children who were randomly assigned to twelve weeks of treatment with omega-3 fatty acids or an identical placebo. In addition to examining the effects of omega-3 fatty acids on hyperactivity and the core features of ASD, one of our main goals was to attempt to identify a biomarker that might identify a subgroup of children who is most likely to benefit from treatment. We therefore examined changes in a panel of 27 plasma cytokines, including IL- 1 and TNF- $\alpha$, as well as changes in inflammatory lipids knows as eicosanoids. To determine whether any improvements in hyperactivity were related to fatty acids, we also examined changes in a panel of 33 serum fatty acids in all subjects. Our prespecified analytical plan was to determine if improvements in hyperactivity were correlated with either baseline levels or changes in the levels of these biomarkers. If the analysis reveals that specific biomarkers are correlated with changes in hyperactivity, it may be possible to recruit a population of children with a specific biochemical profile to enroll in a future study. This type of targeted recruitment may improve the power of studies to identify beneficial effects.

\section{Inflammation: Hyperbaric oxygen therapy}

Hyperbaric oxygen therapy (HBOT) provides 100\% inhaled oxygen to patients at a pressure greater than 1 atmosphere in a pressurized chamber. The treatment increases the oxygen content of plasma, body tissues, and ischemic tissue, and is used in a variety of conditions including closed-head trauma, ischemic brain injury, de- compression sickness, and carbon monoxide poisoning. ${ }^{50}$ Some authors have theorized that HBOT might improve oxygen delivery to the brain and reduce neuroinflammation and oxidative stress, which could improve symptoms of autism. ${ }^{51}$ However, a potential concern is that the delivery of high concentrations of oxygen could actually increase oxidative stress by providing more substrate (oxygen) to create the highly reactive, and potentially damaging, oxygen free radicals. We are not aware of any studies that have examined changes in markers of oxidative stress in children with ASD undergoing HBOT. Two previous small, randomized, controlled trials of HBOT have been conducted in children with ASD, with one reporting improvements in overall symptoms (30\% of children improved on the CGI-Improvement score in the HBOT group versus $8 \%$ in the placebo group), ${ }^{52}$ and the other trial resulted in a finding of no effect. ${ }^{53}$

Because one of the main postulated mechanisms for HBOT improving symptoms of autism is an anti-inflammatory effect, we conducted an open-label clinical trial in 10 children between the ages of 3 and 8 with ASD to determine if there were observable changes in detailed markers of inflammation after treatment. Children were treated with 40 sessions of HBOT, and a panel of 27 plasma cytokines was measured at baseline and after 40 days and 80 days (close-out) of treatment. Parental reports indicated that most children improved with treatment, although this was an uncontrolled study. However, initial analytical results have not identified any changes in the plasma cytokines, suggesting that if there is a beneficial effect to HBOT, it does not occur through anti-inflammatory activity (manuscript in preparation). The children in this study did not have elevated markers of inflammation at baseline, so the therapy might have different effects in children with increased inflammatory states. In addition, the small sample size and the known variability of cytokines provided limited power, and this negative study does not rule out inflammation as a potential mechanism of action of HBOT. Because both inflammation and antioxidant effects are believed to play a role in the potential benefit of HBOT in ASD, future, larger studies should consider including these potential biomarkers to determine if benefits are related to changes in these variables.

\section{Functional brain measures to identify subgroups of children with sensory dysfunction}

Prior studies have suggested that as many as $95 \%$ of children with autism have abnormalities in sensory processing, including problems with under-responsiveness/ seeking sensation, auditory filtering, and tactile sensitivity ${ }^{54}$ In a recent pilot study, investigators in our research group used magnetoencephalography to examine brain responses to various tones in children with ASD com- 
pared to healthy controls (manuscript under preparation), and identified different signal processing in the two groups. Previous studies have found that certain magnetoencephalography signals are associated with auditory and language impairment in $\mathrm{ASD},{ }^{55}$ and others have found that particular brain regions may be unable to generate high-frequency activity that may be necessary for connecting signals to various parts of the brain. ${ }^{56}$ Similarly, other groups have identified psychophysiological measures, such as abnormal eye tracking and pupillary reactivity, which are associated with the fragile $\mathrm{X}$ syndrome.$^{57}$ Both magnetoencephalography and psychophysiological measures may be able to identify specific subgroups of children with sensory processing problems or other manifestations of ASD who might be most likely to benefit from targeted interventions.

\section{WIDE RANGE OF POSSIBLE OUTCOME MEASURES}

One of the major challenges in clinical trials of interventions for ASD is selecting the primary outcome measure. As previously noted, ASD involves three core symptom areas (communication problems, difficulties with social interactions, and restricted/repetitive behaviors), but also commonly involves associated symptoms such as gastrointestinal problems, anxiety and obsessivecompulsive symptoms, behavioral problems such as hyperactivity, irritability, insomnia, and abnormal sensory integration. The list of possible symptoms for any given treatment is therefore extremely long, and there are many different scales and many different methods of assessing response to therapy (e.g., parent, caregiver, teacher evaluations, clinical observations, and structured vs unstructured observations).

Numerous outcome measures are available and have been reviewed previously. ${ }^{58}$ Although certain instruments are commonly used, currently there is no consensus on the set of outcome measures that should be included in all clinical trials. Clearly, it would be desirable to have a standard set of outcomes for all clinical trials of therapies for ASD, because this would enable the comparison of different interventions between studies. Clinical trials would not have to be limited to a standard set of outcomes, but could use these established outcomes in addition to any others that might be of particular interest with a specific intervention. One instrument that is commonly used to assess global function in ASD is the CGI scale, which has subscales for severity and improvement. The improvement subscale ranges from 1 (very much improved) to 7 (very much worse). Although the concept of using a standard global measure of functioning is desirable, the CGI is limited by the relatively "coarse" calibration (i.e., there are only 7 points representing the full range of change from very much improved to very much worse). Similarly, anchors for the CGI scale are not always used (i.e, clinical trials do not always define what specific change in participant symptoms should be coded as a corresponding change in the CGI score). Therefore, an improvement of 2 points in the CGI in one study may not be equivalent to a 2-point improvement in another study.

Another approach to selecting outcome measures in clinical trials of autism might be to use one scale for each core symptom of autism or other area of interest. For example, one might select the CGI for global changes, the Peabody Picture Vocabulary Test and the Expressive Vocabulary Test for changes in communication, the Social Responsiveness Scale for changes in social functioning, and the Aberrant Behavior Checklist for changes in behaviors. However, the number of subscales and outcome measures may become quite large, which then creates problems with multiple comparisons, increasing the likelihood of observing statistically significant changes that are due to chance (unless adjustments are applied for multiple comparisons, which then limits the power to detect changes in the most relevant outcome measures).

An understanding of the underlying biochemical, physiological, and structural changes in ASD may also help with the selection of the large array of potential outcome measures. For example, if inflammation is found to be highly correlated with hyperactivity, then it would make sense to focus on hyperactivity in future studies of anti-inflammatory agents, such as HBOT or omega-3 fatty acids. Similarly, studies evaluating therapies to treat sensory abnormalities might consider using functional brain measures to assess the neural response to certain stimuli before and after interventions. The interventions that are believed to reduce the response to tactile stimulation could focus on quantifying the neural response to standard stimulation.

\section{RECRUITING LARGE SAMPLES FOR CLINICAL TRIALS}

We believe that the largest randomized, controlled trial of autism to date has only enrolled 218 subjects, ${ }^{6}$ and there are only a few studies that enrolled more than 100 patients. The sample sizes are very small compared to studies of interventions in other disorders, such as cardiovascular disease, in which clinical trials commonly involve thousands of patients. Recruitment is complicated by the limited number and scope of registries for children with ASD, the often intensive and time-consuming nature of the assessments (to validate the diagnosis), the behavioral challenges of affected children, and the competing priorities of families who are already burdened with the stress of managing their child. It may also be difficult to evaluate therapies that may take longer to 
produce beneficial effects, because parents may be reluctant to agree to hold other treatments stable for a long period of time. If parents are using many therapies during the course of a study, it may be more difficult to determine if any beneficial effects are caused by the intervention under study. Ongoing efforts to create large, wellcharacterized registries of children with ASD and to create clinical trial networks may improve the ability to enroll larger numbers of children. It may also be possible to use the internet, both to reach more families of children with ASD and to obtain outcome measures of parental reports to interventions, thus simplifying study design, reducing cost, and allowing for larger sample sizes.

\section{CONCLUSION}

The clinical constellation of symptoms that defines ASD likely represents a diverse set of underlying genetic and biochemical abnormalities. Standard randomized, controlled trials, which generally compare the mean response to an intervention versus a control, may be unable to identify therapies that improve only one of the many underlying abnormalities in ASD. A greater understanding of the altered genetic and biochemical pathways may allow for targeted recruitment of subjects with a specific abnormality that is most likely to respond to treatment. In recently completed and ongoing studies of biomedical interventions in ASD, we have found that biomarkers have shed light on possible underlying pathways. Future studies will determine if these biomarkers are useful for targeted recruitment or the prediction of response to therapy.

Acknowledgments: This work was supported by grants from Autism Speaks, the Higgins Family Foundation, The Emch Foundation, and The Taube Foundation (Dr. Bent).

\section{REFERENCES}

1. Autism and Developmental Disabilities Monitoring Network Surveillance Year 2006 Principal Investigators; Centers for Disease Control and Prevention (CDC). Prevalence of autism spectrum disorders - Autism and Developmental Disabilities Monitoring Network, United States, 2006. MMWR Surveill Summ 2009;58: $1-20$.

2. Educating Children with Autism. Washington, DC: National Academy Press, 2001.

3. Ospina MB, Krebs Seida J, Clark B, et al. Behavioural and developmental interventions for autism spectrum disorder: a clinical systematic review. PloS One 2008;3:e3755.

4. McCracken JT, McGough J, Shah B, et al. Risperidone in children with autism and serious behavioral problems. N Engl J Med 2002; 347:314-321.

5. Shea S, Turgay A, Carroll A, et al. Risperidone in the treatment of disruptive behavioral symptoms in children with autistic and other pervasive developmental disorders. Pediatrics 2004;114:e634e641.

6. Marcus RN, Owen R, Kamen L, et al. A placebo-controlled, fixeddose study of aripiprazole in children and adolescents with irritability associated with autistic disorder. J Am Acad Child Adolesc Psychiatry 2009;48:1110-1119.
7. Owen R, Sikich L, Marcus RN, et al. Aripiprazole in the treatment of irritability in children and adolescents with autistic disorder. Pediatrics 2009;124:1533-1540.

8. King BH, Hollander E, Sikich L, et al. Lack of efficacy of citalopram in children with autism spectrum disorders and high levels of repetitive behavior: citalopram ineffective in children with autism. Arch Gen Psychiatry 2009;66:583-590.

9. ACTN Trial: Study of fluoxetine in autism (SOFIA). http://www. autismspeaks.org/science/programs/ctn/index.php\#fluoxetine_study. Accessed April 21, 2009.

10. Harrington JW, Rosen L, Garnecho A, Patrick PA. Parental perceptions and use of complementary and alternative medicine practices for children with autistic spectrum disorders in private practice. J Dev Behav Pediatr 2006;27(2 Suppl):S156-S161.

11. Heuer L, Ashwood P, Schauer J, et al. Reduced levels of immunoglobulin in children with autism correlates with behavioral symptoms. Autism Res 2008;1:275-283.

12. Enstrom AM, Onore CE, Van de Water JA, Ashwood P. Differential monocyte responses to TLR ligands in children with autism spectrum disorders. Brain Behav Immun xxxx;24:64-71.

13. Enstrom AM, Lit L, Onore CE, et al. Altered gene expression and function of peripheral blood natural killer cells in children with autism. Brain, behavior, and immunity. 2009;23:124-133.

14. Enstrom AM, Van de Water JA, Ashwood P. Autoimmunity in autism. Curr Opin Investig Drugs 2009;10:463-473.

15. Ashwood P, Wills S, Van de Water J. The immune response in autism: a new frontier for autism research. J Leukoc Biol 2006; 80:1-15.

16. Chez MG, Dowling T, Patel PB, Khanna P, Kominsky M. Elevation of tumor necrosis factor-alpha in cerebrospinal fluid of autistic children. Pediatr Neurol 2007;36:361-365.

17. Pardo CA, Vargas DL, Zimmerman AW. Immunity, neuroglia and neuroinflammation in autism. International review of psychiatry (Abingdon, England). 2005;17:485-495.

18. Vargas DL, Nascimbene C, Krishnan C, Zimmerman AW, Pardo CA. Neuroglial activation and neuroinflammation in the brain of patients with autism. Ann Neurol 2005;57:67-81.

19. Zimmerman AW, Jyonouchi H, Comi AM, et al. Cerebrospinal fluid and serum markers of inflammation in autism. Pediatr Neurol 2005;33:195-201.

20. Ashwood P, Wakefield AJ. Immune activation of peripheral blood and mucosal CD3 + lymphocyte cytokine profiles in children with autism and gastrointestinal symptoms. J Neuroimmunol 2006;173: $126-134$

21. Chauhan A, Chauhan V, Brown WT, Cohen I. Oxidative stress in autism: increased lipid peroxidation and reduced serum levels of ceruloplasmin and transferrin - the antioxidant proteins. Life Sci 2004;75:2539-2549.

22. James SJ, Cutler P, Melnyk S, et al. Metabolic biomarkers of increased oxidative stress and impaired methylation capacity in children with autism. Am J Clin Nutr 2004;80:1611-1617.

23. Yorbik O, Sayal A, Akay C, Akbiyik DI, Sohmen T. Investigation of antioxidant enzymes in children with autistic disorder. Prostaglandins Leukot Essent Fatty Acids 2002;67:341-343.

24. Mostafa GA, El-Hadidi ES, Hewedi DH, Abdou MM. Oxidative stress in Egyptian children with autism: relation to autoimmunity. J Neuroimmunol 2010;219:114-118.

25. James SJ, Rose S, Melnyk S, et al. Cellular and mitochondrial glutathione redox imbalance in lymphoblastoid cells derived from children with autism. Faseb J 2009;23:2374-2383.

26. Bell JG, Miller D, Macdonald DJ, et al. The fatty acid composition of erythrocyte and plasma polar lipids in children with autism, developmental delay or typically developing controls and the effect of fish oil intake. Br J Nutr 2010;103:1160-1167.

27. Bell JG, MacKinlay EE, Dick JR, MacDonald DJ, Boyle RM, Glen AC. Essential fatty acids and phospholipase A2 in autistic spectrum disorders. Prostaglandins Leukot Essent Fatty Acids 2004; 71:201-204.

28. Meguid NA, Atta HM, Gouda AS, Khalil RO. Role of polyunsaturated fatty acids in the management of Egyptian children with autism. Clin Biochem 2008;41:1044-1048.

29. Vancassel S, Durand G, Barthelemy C, et al. Plasma fatty acid 
levels in autistic children. Prostaglandins Leukot Essent Fatty Acids 2001;65:1-7.

30. Bu B, Ashwood P, Harvey D, King IB, Water JVd, Jin LW. Fatty acid compositions of red blood cell phospholipids in children with autism. Prostaglandins Leukot Essent Fatty Acids 2006;74:215221.

31. Zecavati N, Spence SJ. Neurometabolic disorders and dysfunction in autism spectrum disorders. Curr Neurol Neurosci Rep 2009;9: $129-136$.

32. Oliveira G, Diogo L, Grazina M, et al. Mitochondrial dysfunction in autism spectrum disorders: a population-based study. Dev Med Child Neurol 2005;47:185-189.

33. Weissman JR, Kelley RI, Bauman ML, et al. Mitochondrial disease in autism spectrum disorder patients: a cohort analysis. PloS One 2008;3:e3815.

34. Aldred S, Moore KM, Fitzgerald M, Waring RH. Plasma amino acid levels in children with autism and their families. J Autism Dev Disord 2003;33:93-97.

35. Niederhofer H. Glutamate antagonists seem to be slightly effective in psychopharmacologic treatment of autism. J Clin Psychopharmacol 2007;27:317-318.

36. Fatemi SH, Halt AR, Stary JM, Kanodia R, Schulz SC, Realmuto GR. Glutamic acid decarboxylase 65 and $67 \mathrm{kDa}$ proteins are reduced in autistic parietal and cerebellar cortices. Biol Psychiatry 2002;52:805-810.

37. Polleux F, Lauder JM. Toward a developmental neurobiology of autism. BMC Complement Altern Med 2004;10:303-317.

38. Bertoglio K, James JS, Deprey L, Brule N, Hendren RL. Pilot study of the effect of methyl B12 treatment on behavioral and biomarker measures in children with autism. BMC Complement Altern Med 2010;16:1-6.

39. Golub TR, Slonim DK, Tamayo P, et al. Molecular classification of cancer: class discovery and class prediction by gene expression monitoring. Science 1999;286:531-537.

40. Alizadeh AA, Staudt LM. Genomic-scale gene expression profiling of normal and malignant immune cells. Curr Opin Immunol 2000; $12: 219-225$.

41. Hedenfalk I, Duggan D, Chen Y, et al. Gene-expression profiles in hereditary breast cancer. N Engl J Med 2001;344:539-548.

42. Staudt LM. Gene expression physiology and pathophysiology of the immune system. Trends Immunol 2001;22:35-40.

43. Simons JW. Genomic medicines for oncology: early lessons. J Med Assoc Ga. Winter-Spring 2003;92:35-38.

44. Chin KV, Alabanza L, Fujii K, et al. Application of expression genomics for predicting treatment response in cancer. Ann N Y Acad Sci 2005;1058:186-195.

45. Carroll WL, Bhojwani D, Min DJ, Moskowitz N, Raetz EA. Childhood acute lymphoblastic leukemia in the age of genomics. Pediatr Blood Cancer 2006;46:570-578.

46. Green VA, Pituch KA, Itchon J, Choi A, O'Reilly M, Sigafoos J. Internet survey of treatments used by parents of children with autism. Res Dev Disabil 2006;27:70-84.

47. Amminger GP, Berger GE, Schafer MR, Klier C, Friedrich MH, Feucht M. Omega-3 fatty acids supplementation in children with autism: a double-blind randomized, placebo-controlled pilot study. Biol Psychiatry 2007;61:551-553.

48. Simopoulos AP. Omega-3 fatty acids in inflammation and autoimmune diseases. J Am Coll Nutr 2002;21:495-505.

49. Young G, Conquer J. Omega-3 fatty acids and neuropsychiatric disorders. Reprod Nutr Dev 2005;45:1-28.

50. Rossignol DA, Rossignol LW. Hyperbaric oxygen therapy may improve symptoms in autistic children. Med Hypotheses 2006;67: 216-228.

51. Rossignol DA. Hyperbaric oxygen therapy might improve certain pathophysiological findings in autism. Med Hypotheses 2007;68: $1208-1227$.

52. Rossignol DA, Rossignol LW, Smith S, et al. Hyperbaric treatment for children with autism: a multicenter, randomized, double-blind, controlled trial. BMC Pediatr 2009;9:21.

53. Granpeesheh D, Tarbox J, Dixon DR, Wilke AE, Allen MS, Bradstreet JJ. Randomized trial of hyperbaric oxygen therapy for children with autism. Res Autism Spectr Disord 2010;4:268-275.

54. Tomchek SD, Dunn W. Sensory processing in children with and without autism: a comparative study using the short sensory profile. Am J Occup Ther 2007;61:190-200.

55. Roberts TP, Schmidt GL, Egeth M, et al. Electrophysiological signatures: magnetoencephalographic studies of the neural correlates of language impairment in autism spectrum disorders. Int J Psychophysiol 2008;68:149-160.

56. Wilson TW, Rojas DC, Reite ML, Teale PD, Rogers SJ. Children and adolescents with autism exhibit reduced MEG steady-state gamma responses. Biol Psychiatry 2007;62:192-197.

57. Farzin F, Rivera SM, Hessl D. Brief report: visual processing of faces in individuals with fragile $X$ syndrome: an eye tracking study. J Autism Dev Disord 2009;39:946-952.

58. Aman MG, Novotny S, Samango-Sprouse C, et al. Outcome measures for clinical drug trials in autism. CNS Spectrums 2004;9:36-47. 